\title{
Embryonal Rhabdomyosarcoma Presenting as Lung Metastasis in an Adult: An Uncommon Presentation
}

\author{
Rabia Taseer ${ }^{1,2}$, Tabeer T. Ahmed ${ }^{3}$ \\ 1. Histopathology, Shiekh Zaid Hospital, Lahore, PAK 2. Histopathology, Obeid Specialized Hospital, Riyadh, SAU 3. \\ Internal Medicine, Combined Military Hospital (CMH) Lahore Medical College and Institute of Dentistry, Lahore, PAK
}

Corresponding author: Rabia Taseer, rabiataseer1@gmail.com

\begin{abstract}
Rhabdomyosarcoma (RMS) is one of the most common soft tissue sarcomas of adolescents and young adults. Histologically, rhabdomyosarcoma is classified into embryonal, alveolar, pleomorphic, and spindle cell/sclerosing rhabdomyosarcomas with further subcategorization. More than $50 \%$ of embryonal rhabdomyosarcoma occur within head and neck. The retroperitoneum and pelvis are less common sites of involvement. Embryonal rhabdomyosarcomas affect mainly, but not exclusively, children between birth and 15 years of age. Alveolar rhabdomyosarcoma tends to affect older patients. The usual metastatic sites include lung, lymph nodes, and bone marrow. We are presenting a case of a 25 -year-old male patient with symptoms of breathlessness, easy fatigability, and weight loss. On chest imaging, there were multiple lung nodules. A primary diagnosis of undifferentiated malignant neoplasm was made on lung biopsy. On immunohistochemistry, the malignant cells were positive for myogenin, myoblast determination protein 1 (MyoD1), and desmin. They were negative for neuroendocrine, germ cell, epithelial, melanocytic, and lymphoid markers. Further workup showed an abdominopelvic retroperitoneal mass on abdominal computed tomography (CT) scan. The biopsy on the retroperitoneal mass showed similar morphology and immunohistochemical profile. Unfortunately, the patient's condition deteriorated rapidly in the following weeks, and he passed away.
\end{abstract}

Review began 02/10/2021 Review ended 02/18/2021 Published 02/24/2021

() Copyright 2021

Taseer et al. This is an open access article distributed under the terms of the Creative Commons Attribution License CC-BY 4.0., which permits unrestricted use, distribution, and reproduction in any medium, provided the original author and source are credited.
Categories: Pathology, Radiology, Oncology

Keywords: rhabdomyosarcoma, embryonal, myogenin, myo d1, retroperitoneal, lung metastasis

\section{Introduction}

Rhabdomyosarcoma (RMS) is one of the most common soft tissue sarcomas of adolescents and young adults. It accounts for an estimated $4.5 \%$ of all childhood cancers, with an incidence of six cases per one million annually [1]. Histologically, rhabdomyosarcoma is classified into embryonal, alveolar, pleomorphic, and spindle cell/sclerosing rhabdomyosarcomas with further subcategorization [2,3]. More than $50 \%$ of embryonal rhabdomyosarcomas occur within head and neck [4]. Retroperitoneum and abdomen are less common sites [5]. It has been observed that rhabdomyosarcoma can metastasize to lymph nodes, bones, and lungs. However, this is more common in cases of alveolar rhabdomyosarcoma as compared to embryonal subtype [6]. We present a case of a 25 -year-old male patient who presented with lung metastasis from a retroperitoneal embryonal rhabdomyosarcoma.

\section{Case Presentation}

A 25 -year-old male patient presented to the chest clinic with easy fatigability and breathlessness for more than a month. He had a history of weight loss. The chest x-ray revealed right-sided pleural effusion (Figure 1A). On chest computed tomography (CT), there were multiple bilateral lung nodules and a 7-cm mass (with heterogeneous enhancement and non-enhancing possibly necrotic components) on the right side. There were pleural nodules (Figure $1 B$ ). A lung biopsy was performed. The histopathology department received multiple fragments of tissue in formalin; hematoxylin- and eosin-stained sections showed fragments of tissue with varying cellularity. There were densely packed, hypercellular areas (Figures 2A, 2B) and loosely textured, myxoid areas (Figures 3A, 3B). Neoplastic cells were round to oval with pleomorphic hyperchromatic nuclei. Larger atypical cells were also seen. There were no cross striations or poorly differentiated round cells. 


\section{Cureus}

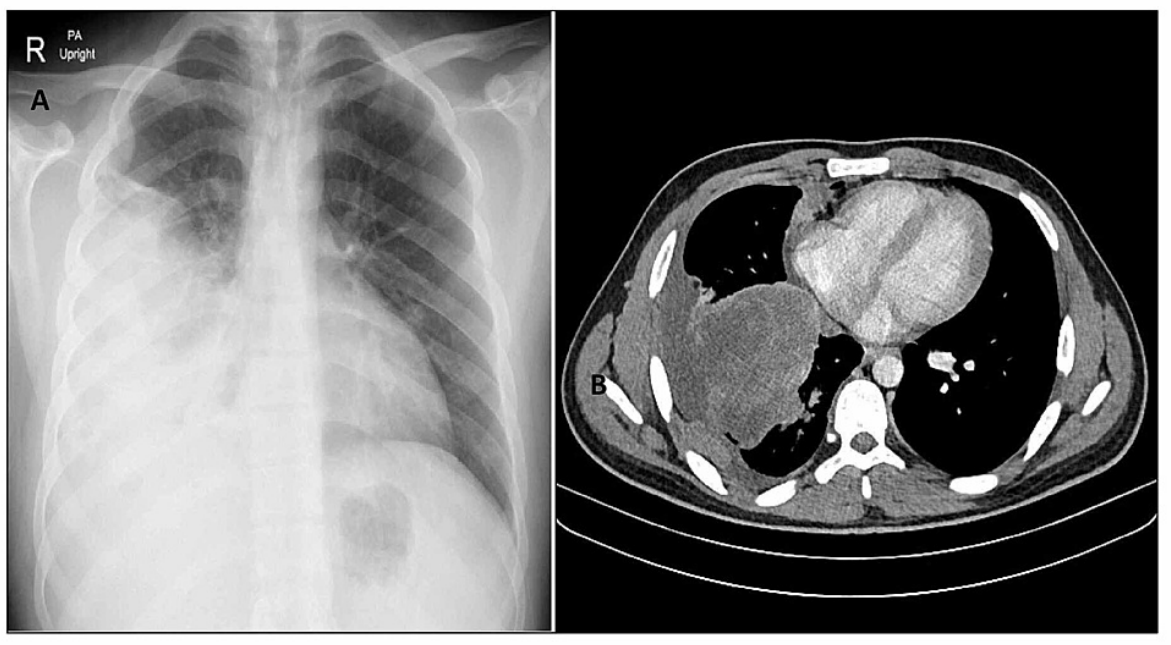

FIGURE 1: (A) Chest $x$-ray: right middle and lower lobe mass with rightsided pleural effusion. (B) Chest CT: right lower lobe mass with multiple smaller bilateral masses.

CT, Computed tomography.
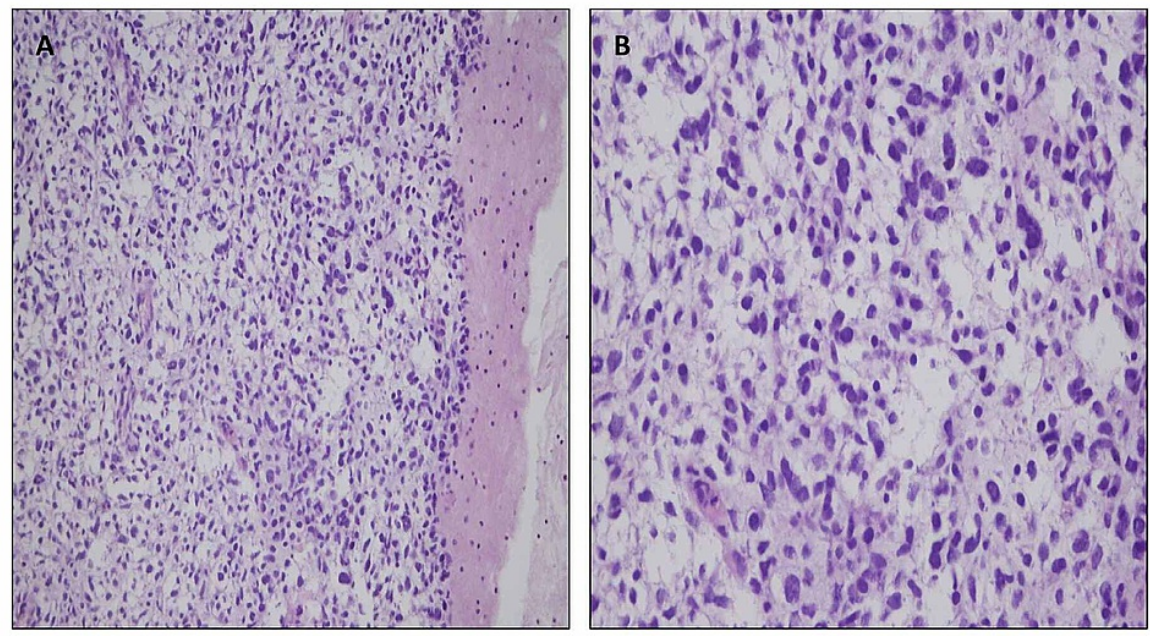

FIGURE 2: Hematoxylin and eosin (H\&E) staining: cellular neoplasm showing pleomorphic cells with varying cellularity and myxoid background. (A) 20x (medium power) and (B) 40x (high power). 


\section{Cureus}

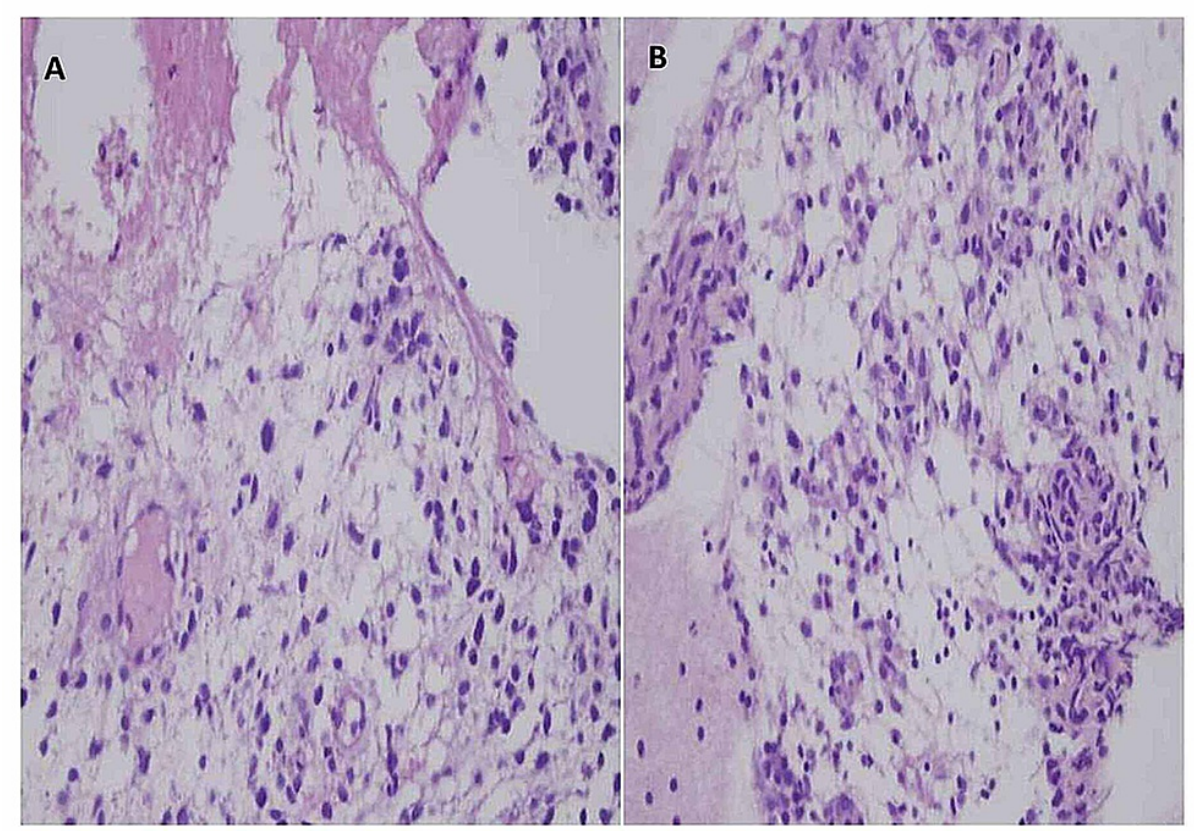

FIGURE 3: Hematoxylin and eosin (H\&E) staining showing hypocellular areas and myxoid stroma. (A) 40X (high power) and (B) 20X (medium power).

A primary diagnosis of undifferentiated malignant neoplasm was made with a wide differential diagnosis. We considered neuroblastoma, lymphoma, and germ cell tumor. Immunohistochemistry with neuronspecific enolase, chromogranin, synaptophysin, Sal-like protein 4 (SALL4), and CD45 was done to rule out these diagnoses. Despite the unusual age bracket, the morphology was strongly suggestive of embryonal rhabdomyosarcoma, therefore myoregulatory proteins (myogenin and MyoD1) and desmin were added to the immunopanel. Cytokeratin and S100 were added to rule out the less likely differentials of carcinoma and malignant melanoma.

On immunohistochemistry, the tumor was negative for neuroendocrine, germ cell, epithelial, melanocytic, and lymphoid markers (Figure 4). Both myogenin and MyoD1 showed nuclear positivity in malignant cell. Myogenin expression was seen in less than $20 \%$ of tumor cells, and the intensity of staining was mild (Figure 5A). MyoD1 was more diffusely expressed (Figure 5B). Desmin showed cytoplasmic positivity (Figure 6). Based on immunohistochemistry, a diagnosis of metastatic rhabdomyosarcoma was made. The pattern of immunostain expression and morphology was considered to be consistent with embryonal rhabdomyosarcoma. After the histopathological diagnosis of embryonal rhabdomyosarcoma, the patient was evaluated by oncology department. An abdominopelvic CT was done, which showed a retroperitoneal abdominopelvic mass measuring $12.5 \mathrm{~cm}$ in maximum dimension, displacing rectum and urinary bladder (Figure 7). The biopsy from the retroperitoneal mass showed similar histology (Figure $8 \mathrm{~A}$ ). On immunohistocemistry the neoplastic cells showed diffuse MyoD1 positivity. They were focally positive for myogenin (Figures $8 B, 8 \mathrm{C}$ ). Desmin was positive in malignant cells. Mouse double minute 2 homolog (MDM2) and caldesmon were negative, ruling out dedifferentiated liposarcoma and leiomyosarcoma. 


\section{Cureus}
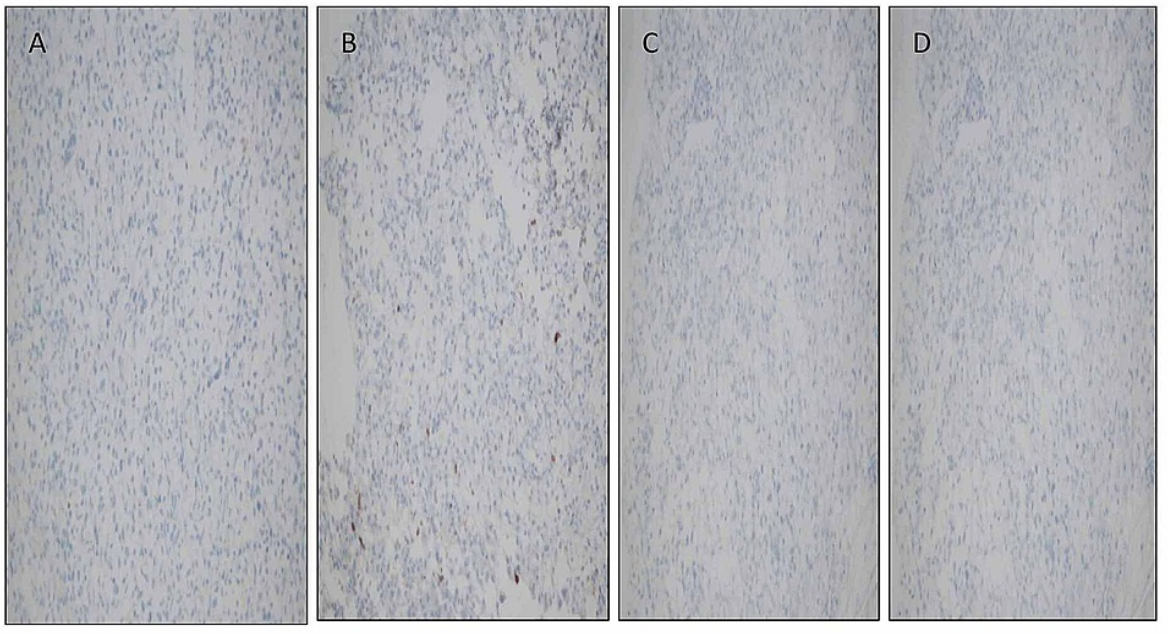

FIGURE 4: Immunohistochemical stains: (A) chromogranin, (B) S100, (C) SALL4, and (D) pan cytokeratin - negative in malignant cells.

SALL4, Sal-like protein 4.
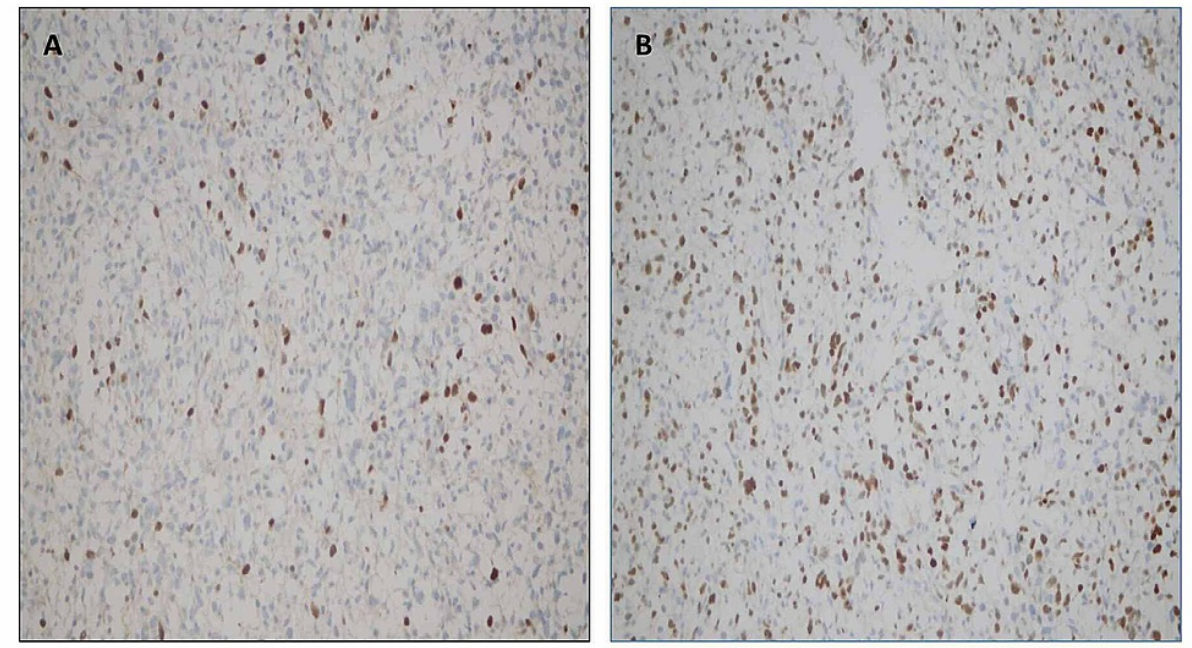

FIGURE 5: Immunohistochemistry - (A) Myogenin: positive in around $20 \%$ of tumor cells. (B) MyoD1: diffuse positivity in malignant cells. 


\section{Cureus}
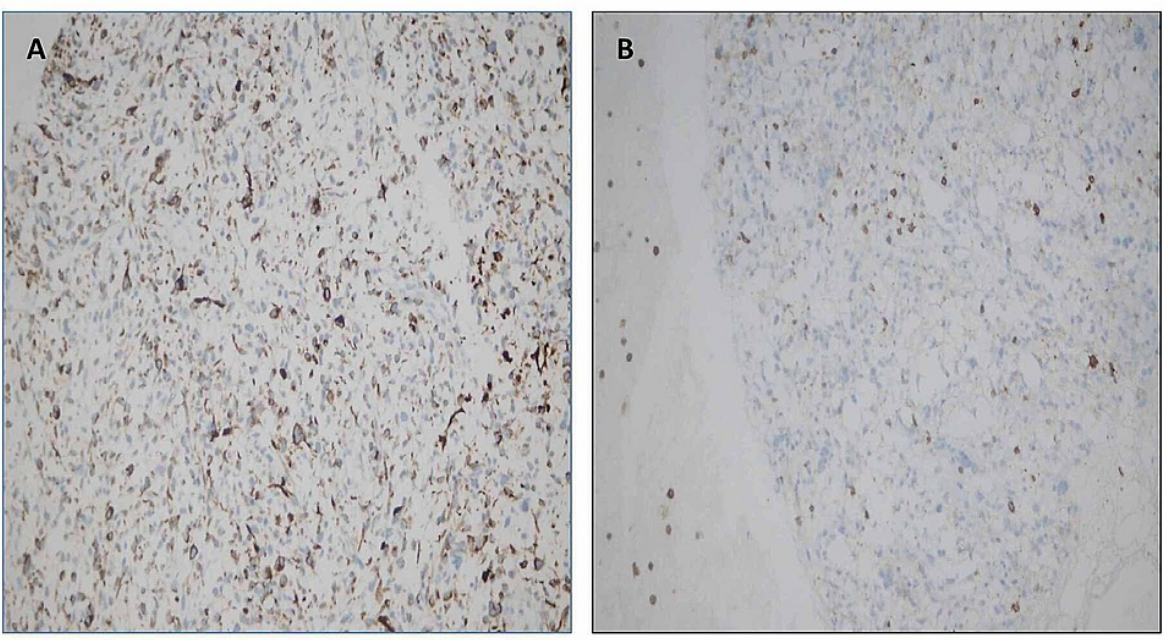

FIGURE 6: Immunohistochemistry - (A) Desmin: cytoplasmic positivity and large malignant cells. (B) CD45: negative in malignant cells.

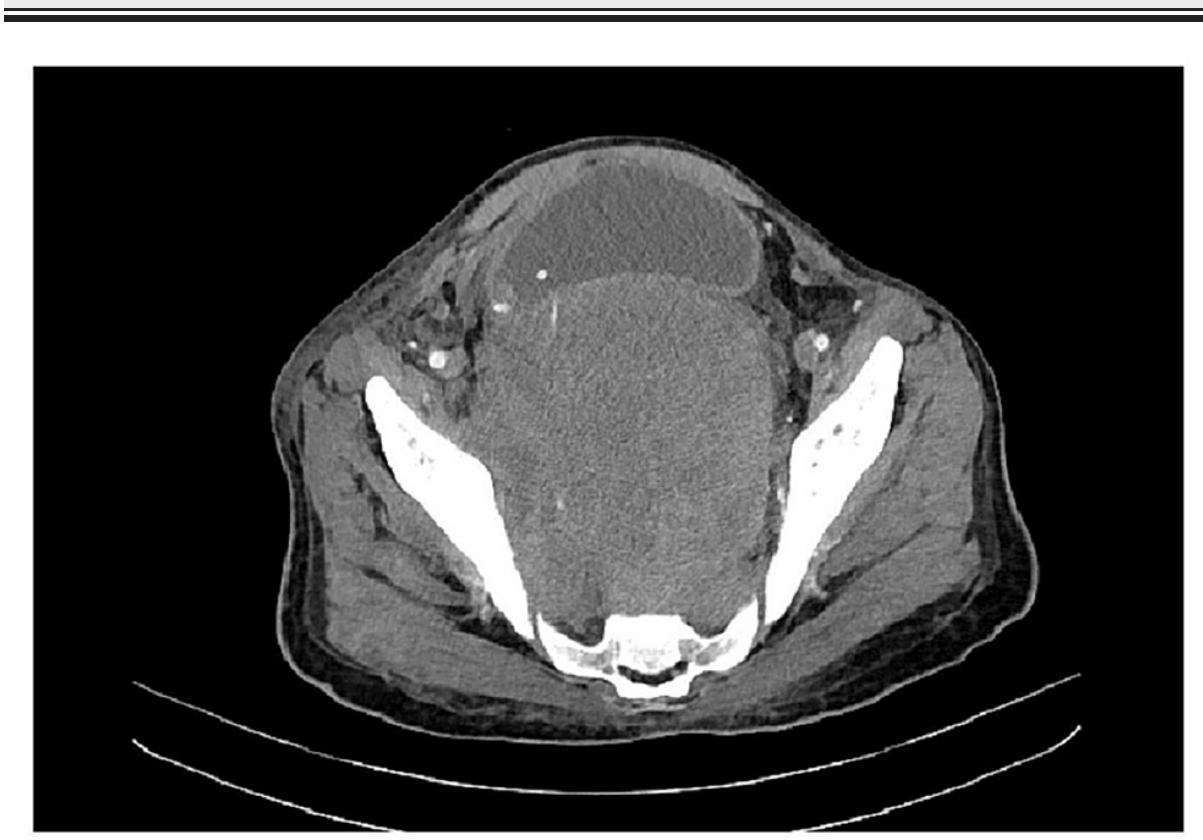

FIGURE 7: Pelvic CT scan: a retroperitoneal mass measuring $12.5 \mathrm{~cm}$ occupying the pelvic cavity.

CT, Computed tomography. 


\section{Cureus}
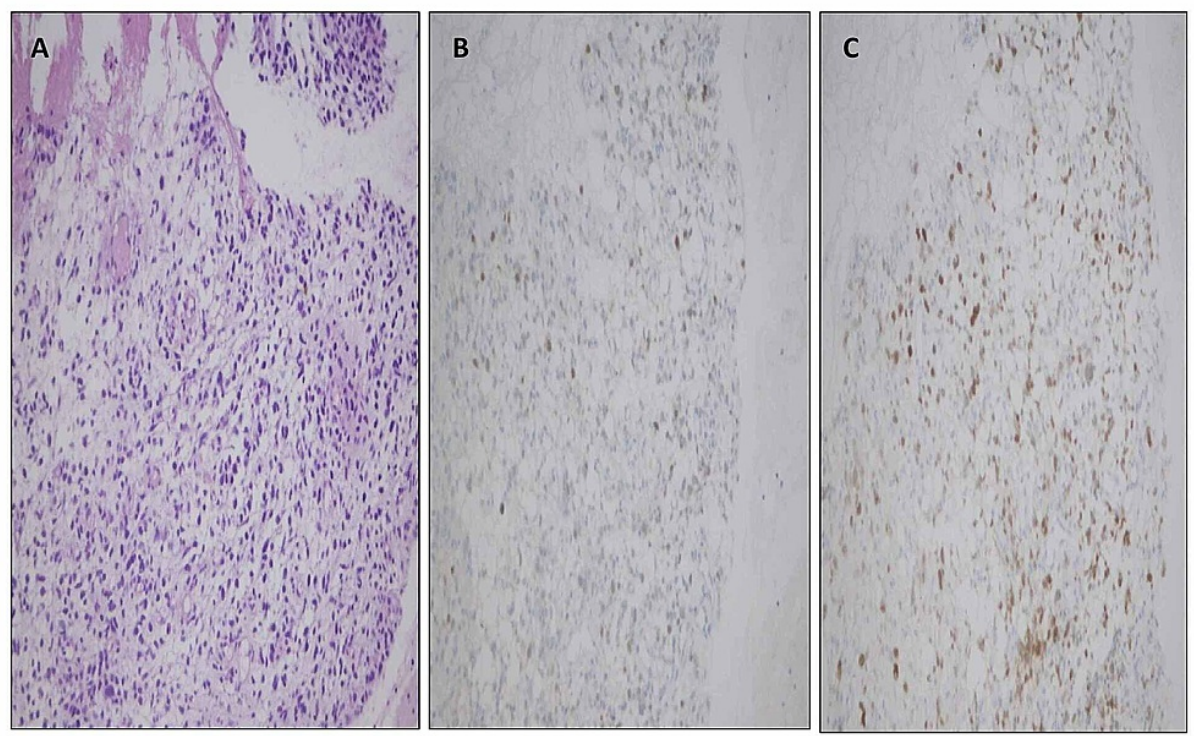

\section{FIGURE 8: Biopsy pelvic mass - (A) hematoxylin and eosin stain, (B) myogenin immunostain, and (C) MyoD1 immunostain.}

On follow-up, the patient's condition deteriorated rapidly within weeks following the diagnosis, and he passed away.

\section{Discussion}

Rhabdomyosarcoma is a high-grade malignant neoplasm, with a morphologic appearance mimicking that of developing skeletal muscle. It accounts for $4.5 \%$ of all childhood cancers. The annual incidence is around six cases per one million per year [1,2]. Histologically, rhabdomyosarcoma is classified into embryonal rhabdomyosarcoma (botryoides variant and anaplastic variant), alveolar rhabdomyosarcoma (solid variant and anaplastic variant), pleomorphic rhabdomyosarcoma, and spindle cell/sclerosing rhabdomyosarcoma. Recent studies have significantly impacted this classification with the emergence of three distinct new subtypes of rhabdomyosarcomas, namely rhabdomyosarcoma with MyoD1 mutations, rhabdomyosarcoma with TFCP2 fusions, and rhabdomyosarcoma with VGLL2/NCOA2 fusions [3].

More than $50 \%$ of embryonal rhabdomyosarcomas occur within head and neck region [4].

Retroperitoneum and abdomen are less common sites [5]. In the present case the primary tumor site was retroperitoneal. Each of the rhabdomyosarcoma subtypes occurs in a characteristic age group. Embryonal rhabdomyosarcomas affects mainly children between birth and 15 years of age; it is less common in older patients. On the other hand, alveolar rhabdomyosarcoma tends to affect older patients, with peak ages of $10-25$ years $[6,7]$. The prognosis of embryonal rhabdomyosarcoma is poorer in older age group as compared to children [8]. Our case was an older patient. A large number of patients with rhabdomyosarcoma are considered high-risk patients as they show distant metastatic disease, involving lymph nodes, bone marrow, and lungs. But metastasis has been observed more commonly in patients with alveolar as compared to embryonal rhabdomyosarcoma [9]. In our case, distant metastasis (in lung) was observed with embryonal subtype.

Morphologically, embryonal rhabdomyosarcomas are composed of primitive mesenchymal cells that show variable degrees of skeletal muscle differentiation. They are moderately cellular, but in the typical pattern they often contain both hypo- and hypercellular areas with a loose, myxoid stroma sheets of small, stellate, spindled, or round cells with scant or deeply eosinophilic cytoplasm and eccentric, small oval nuclei with a light chromatin pattern and inconspicuous nucleoli. Feature of rhabdomyoblastic differentiation (so called "strap" cells) can be seen [10]. On hematoxylin and eosin (H\&E) stain, our case showed varying cellularity, alternating between densely packed, hypercellular areas and loosely textured, myxoid areas. Neoplastic cells were round to oval with pleomorphic hyperchromatic nuclei. Larger atypical cells were also seen (Figures 2, 3).There were no cross striations. No poorly differentiated round cells were seen.

Since our case was a metastatic lesion in the lung, we would not rely solely on morphology as it can be deceptive in metastatic disease. In cases of less differentiated tumors, it has been seen that the best method to detect rhabdomyoblastic differentiation of the sarcoma is the demonstration of expression of MyoD1 protein and myogenin. Dias et al. demonstrated that positive nuclear staining in both markers is an important diagnostic criterion for rhabdomyosarcoma. It is considered the gold standard in differential diagnosis of this tumor from other neoplasms. Alveolar rhabdomyosarcomas shows three times more 
myogenin expression than embryonal rhabdomyosarcomas. According to their data, staining for myogenin is a simple, rapid, and accurate tool for differentiating between embryonal and alveolar rhabdomyosarcomas [11]. Similarly, Rudzinski et al. and Rakhi et al. have demonstrated in separate studies that embryonal rhabdomyosarcoma shows focal myogenin immunostaining pattern, whereas myogenin expression is more diffuse in alveolar rhabdomyosarcoma. MyoD1, on the other hand, is more diffusely expressed in embryonal rhabdomyosarcoma [12,13]. Our case showed a nuclear positivity for both myoregulatory proteins (myogenin and MyoD1), but myogenin expression was seen in less than 20\% of tumor cells, and the intensity of staining was mild. MyoD1 expression was more diffuse in our case (Figure 5). It is important to mention here that these antibodies also provide an alternative to molecular methods for identification of fusion-positive rhabdomyosarcomas [14,15]. Cytoplasmic expression of vimentin and desmin is expected to be positive in poor or undifferentiated cells in embryonal rhabdomyosarcomas [15]. Our case showed cytoplasmic positivity for desmin in the large pleomorphic cells. On the basis of immunohistochemistry and morphology, a diagnosis of metastatic embryonal rhabdomyosarcoma was made.

The patient was referred to the oncology department. An abdominal CT showed a retroperitoneal abdominopelvic mass. According to the literature, the most common retroperitoneal sarcoma is dedifferentiated liposarcoma followed by leiomyosarcoma ( $45 \%$ and $21 \%$, respectively). Rhabdomyosarcoma is far less common in retroperitoneum $[16,17]$. On H\&E, the morphology of retroperitoneal mass was similar to the lung mass. On immunohistochemistry, the neoplastic cells showed diffuse MyoD1 positivity. They were focally positive for myogenin and desmin. MDM2 and caldesmon were negative, ruling out dedifferentiated liposarcoma and leiomyosarcoma.

Unfortunately, the patient's condition deteriorated rapidly after the diagnosis, and he passed away two months after his presentation to the outpatient department.

\section{Conclusions}

Embryonal rhabdomyosarcoma is considered to be more common in ages from birth to 15 years. The most common site for this malignancy is head and neck; retroperitoneum and abdomen are less common sites. Our case is an example of a retroperitoneal embryonal rhabdomysarcoma in a 25-year-old patient. The primary presentation in this case was with respiratory symptoms due to lung metastasis.

\section{Additional Information \\ Disclosures}

Human subjects: Consent was obtained or waived by all participants in this study. Conflicts of interest: In compliance with the ICMJE uniform disclosure form, all authors declare the following: Payment/services info: All authors have declared that no financial support was received from any organization for the submitted work. Financial relationships: All authors have declared that they have no financial relationships at present or within the previous three years with any organizations that might have an interest in the submitted work. Other relationships: All authors have declared that there are no other relationships or activities that could appear to have influenced the submitted work.

\section{References}

1. Dasgupta R, Fuchs J, Rodeberg D: Rhabdomyosarcoma. Semin Pediatr Surg. 2016, 25:276-283. 10.1053/j.sempedsurg.2016.09.011

2. Skapek SX, Ferrari A, Gupta AA, et al.: Rhabdomyosarcoma. Nat Rev Dis Primers. 2019, 1038:41572-018. 10.1038/s41572-018-0051-2

3. Leiner J, Le Loarer F: The current landscape of rhabdomyosarcomas: an update . Virchows Arch. 2020, 476:97-108. 10.1007/s00428-019-02676-9

4. Raney RB, Walterhouse DO, Meza JL, et al.: Results of the Intergroup Rhabdomyosarcoma Study Group D9602 protocol, using vincristine and dactinomycin with or without cyclophosphamide and radiation therapy, for newly diagnosed patients with low-risk embryonal rhabdomyosarcoma: a report from the Soft Tissue Sarcoma Committee of the Children's Oncology Group. J Clin Oncol. 2011, 29:1312-8. 10.1200/jco.2010.30.4469

5. Huang CJ: Rhabdomyosarcoma involving the genitourinary organs, retroperitoneum, and pelvis . J Pediatr Surg. 1986, 2:101-7. 10.1016/s0022-3468(86)80058-6

6. Stock N, Chibon F, Binh MB, et al.: Adult-type rhabdomyosarcoma: analysis of 57 cases with clinicopathologic description, identification of 3 morphologic patterns and prognosis. Am J Surg Pathol. 2009, 33:1850-9. 10.1097/pas.0b013e3181be6209

7. Rudzinski ER, Anderson JR, Chi YY, et al.: Histology, fusion status, and outcome in metastatic rhabdomyosarcoma: a report from the Children's Oncology Group. Pediatr Blood Cancer. 2017, 64:12. 10.1002/pbc. 26645

8. Hibbitts E, Chi YY, Hawkins DS, et al.: Refinement of risk stratification for childhood rhabdomyosarcoma using FOXO1 fusion status in addition to established clinical outcome predictors: a report from the Children's Oncology Group. Cancer Med. 2019, 8:6437-6448. 10.1002/cam4.2504

9. Wexler LH: Metastatic rhabdomyosarcoma: still room for improvement . J Clin Oncol. 2016, 10:105-6. $10.1200 /$ jco.2015.64.3395 


\section{Cureus}

10. Dziuba I, Kurzawa P, Dopierała M, Larque AB, Januszkiewicz-Lewandowska D: Rhabdomyosarcoma in children - current pathologic and molecular classification. Pol J Pathol. 2018, 69:20-32.

10.5114/pjp.2018.75333

11. Dias P, Chen B, Dilday B, Palmer H, et al.: Strong immunostaining for myogenin in rhabdomyosarcoma is significantly associated with tumors of the alveolar subclass. Am J Pathol. 2000, 2:399-408. 10.1016/s00029440(10)64743-8

12. Rudzinski ER, Teot LA, Anderson JR, et al.: Dense pattern of embryonal rhabdomyosarcoma, a lesion easily confused with alveolar rhabdomyosarcoma: a report from the Soft Tissue Sarcoma Committee of the Children's Oncology Group. Am J Clin Pathol. 2013, 140:82-90. 10.1309/ajcpa1wn7arpcmkq

13. Rekhi B, Gupta C, Chinnaswamy G, Qureshi S, Vora T, Khanna N, Laskar S: Clinicopathologic features of 300 rhabdomyosarcomas with emphasis upon differential expression of skeletal muscle specific markers in the various subtypes: a single institutional experience. Ann Diagn Pathol. 2018, 36:50-60.

10.1016/j.anndiagpath.2018.07.002

14. Rudzinski ER, Anderson JR, Lyden ER, et al.: Myogenin, AP2 $\beta$, NOS-1, and HMGA2 are surrogate markers of fusion status in rhabdomyosarcoma: a report from the soft tissue sarcoma committee of the Children's Oncology Group. Am J Surg Pathol. 2014, 5:654-9. 10.1097/pas.0000000000000195

15. Morotti RA, Nicol KK, Parham DM, et al.: An immunohistochemical algorithm to facilitate diagnosis and subtyping of rhabdomyosarcoma: the Children's Oncology Group experience. Am J Surg Pathol. 2006, 30:962-968. 10.1097/00000478-200608000-00005

16. Pham V, Henderson-Jackson E, Doepker MP, et al.: Practical issues for retroperitoneal sarcoma. Cancer Control. 2016, 23:249-64. 10.1177/107327481602300308

17. Wei S, Henderson-Jackson E, Qian X, Bui MM: Soft tissue tumor immunohistochemistry update: illustrative examples of diagnostic pearls to avoid pitfalls. Arch Pathol Lab Med. 2017, 8:1072-1091. 10.5858/arpa.20160417-ra 